Portland State University

PDXScholar

$11-1-1981$

\title{
Massive neutrinos and the stellar stopping power via the neutrino magnetic moment
}

\author{
P.T. Leung \\ Portland State University \\ M. L. Rustgi \\ J. E. Turner \\ Werner Brandt
}

Follow this and additional works at: https://pdxscholar.library.pdx.edu/phy_fac

Part of the Physics Commons

Let us know how access to this document benefits you.

\section{Citation Details}

Rustgi, M. L., Leung, P. T., Turner, J. E., \& Brandt, W. W. (1981). Massive neutrinos and the stellar stopping power via the neutrino magnetic moment. Physical Review A (General Physics), 24(5), 2425-2430.

This Article is brought to you for free and open access. It has been accepted for inclusion in Physics Faculty Publications and Presentations by an authorized administrator of PDXScholar. Please contact us if we can make this document more accessible: pdxscholar@pdx.edu. 


\title{
Massive neutrinos and the stellar stopping power via the neutrino magnetic moment
}

\author{
M. L. Rustgi \\ Health and Safety Research Division, Oak Ridge National Laboratory, Oak Ridge, Tennessee 37830. \\ and Department of Physics, State University of New York, Buffalo, New York 14260 \\ P. T. Leung \\ Department of Physics, State University of New York, Buffalo, New York 14260 \\ J. E. Turner \\ Health and Safety Research Division, Oak Ridge National Laboratory, Oak Ridge, Tennessee 37830 \\ Werner Brandt* \\ Sektion Physik, Universität München, 8008 München 40, Germany \\ (Received 24 December 1980)
}

\begin{abstract}
An exact expression is derived within the high- $Q$ approximation for the spectrum of energy losses of neutrinos interacting via their magnetic moment with electrons. A formula for the stopping power of matter for neutrinos is derived and applied to the study of the solar neutrino anomaly. The total inelastic cross section is given. An upper limit is deduced for the neutrino magnetic moment from the antineutrino spectrum as recently parametrized by Lee. It confirms the bound reported by Cowan and Reines. The distance for stellar neutrinos to thermalize by the interactions studied here, through encounters with stars is found to exceed the radius of the universe by many orders of magnitude. Stellar neutrinos should thus retain the energy given them at their creation and, rather than cluster around galaxies, permeate the universe uniformly.
\end{abstract}

\section{INTRODUCTION}

Ever since Pauli ${ }^{1}$ postulated its existence, the neutrino has remained one of the most intriguing particles in physics. The unexpectedly low counting rate for solar neutrinos, as deduced from measurements with ${ }^{37} \mathrm{Cl}$, has renewed interest in recent times. ${ }^{2}$ One hypothesis to account for this finding holds that solar neutrinos lose a substantial amount of energy in collisions with electrons during their escape from the sun. Since the ${ }^{37} \mathrm{Cl}$ reaction has a threshold of $0.814 \mathrm{MeV}$ and the relevant neutrino energy spectrum from the CNO solar cycle is predicted to peak near $1 \mathrm{MeV}$, the anomaly could be explained through an energy loss by the neutrinos of some $0.2 \mathrm{MeV}$ during their escape from the sun. To explore this possibility, a number of authors have investigated the energy transfer via the magnetic-moment interaction between a neutrino and the electron. ${ }^{3-6}$ These analyses use the energy-loss formulas derived by Bethe ${ }^{7}$ based on the relativistically invariant electron-neutrino electromagnetic interaction form proposed by Carlson and Oppenheimer. ${ }^{8}$ In this note we reexamine the inelastic scattering of neutrinos by electrons via the neutrino magnetic moment. Using a new formula, we discuss the role of small energy losses that arise in calculating the mean rate of energy loss for neutrinos (stopping power). Numerical calculations are carried out for the energy loss from stopping power theory as the neutrino travels from the center of the sun to its surface for a given value of the neutrino mass and magnetic moment. The general conclusion remains that the electromagnetic force alone is unlikely to account for the solar neutrino anomaly. The total ionization cross section as well as the upper limit of the neutrino magnetic moment are examined. An estimate of the thermalization distance for stellar neutrinos is also made, and some cosmological implications are suggested. ${ }^{9}$ 


\section{INELASTIC SCATTERING CROSS SECTION}

For the neutrino, the interaction for the $\mu$ th component $(\mu=0,1,2,3)$ of the current of a neutral, structureless, spin- $\frac{1}{2}$ particle may be written as

$$
J_{\mu}=2 M \lambda\left\langle\overrightarrow{\mathrm{p}}^{\prime}\left|\frac{i \gamma_{0} \sigma_{\mu \nu} q_{v}}{2 M}\right| \overrightarrow{\mathrm{p}}\right\rangle,
$$

where summation over $v$ is implied. Here $M$ and $\lambda$ are the neutrino rest mass and magnetic moment. The components of the energy-momentum-transfer four vector are given by $q=\left(E-E^{\prime}, \overrightarrow{\mathrm{p}}-\overrightarrow{\mathrm{p}}^{\prime}\right)$ where $E$ and $E^{\prime}$ are the neutrino energy before and after collision and $\overrightarrow{\mathrm{p}}$ and $\overrightarrow{\mathrm{p}}^{\prime}$ are the corresponding momenta (eigenstates $|\vec{p}\rangle$ and $\left|\vec{p}^{\prime}\right\rangle$ ). For the Planck constant and the speed of light, we set $\hbar=c=1$; these quantities will be reinstated at the end. The Dirac matrices $\gamma_{\mu}$ satisfy the anticommutation relations

$$
\gamma_{\mu} \gamma_{v}+\gamma_{v} \gamma_{\mu}=2 g_{\mu v}
$$

where $g_{00}=1$ and $g_{i j}=-\delta_{i j}$ when $i, j=1,2,3$, and $\delta_{i j}$ is the Kronecker $\delta$. The operator $\sigma_{\mu \nu}$ in Eq. (1) stands for the commutator

$$
\sigma_{\mu \nu}=i\left[\gamma_{\mu}, \gamma_{v}\right] / 2
$$

We treat the problem in the so-called "high- $Q$ " approximation, in which the struck electron is assumed to be initially free and at rest. The electron then receives all of the energy and momentum lost by the neutrino. The kinetic energy $Q=E-E^{\prime}$ acquired by the electron is then given by

$$
\overrightarrow{\mathrm{q}}^{2}=Q(Q+2 m)
$$

where $m$ is the electron mass. Physically, this approximation is valid when $Q$ is large compared with the binding energy of the electron.

Employing Eq. (1) and carrying out further manipulations, we obtain the differential cross section for the scattering of unpolarized neutrinos for given $E$ as follows:

$$
\begin{aligned}
\frac{d \sigma}{d \Omega}=-\frac{(e \lambda)^{2}\left|\overrightarrow{\mathrm{p}}^{\prime}\right|}{q^{2}|\overrightarrow{\mathrm{p}}|} & \left\{\left[\left(E+E^{\prime}\right) F_{n}-\left(\overrightarrow{\mathrm{p}}+\overrightarrow{\mathrm{p}}^{\prime}\right) \cdot \overrightarrow{\mathrm{G}}_{n}\right]^{2}\right. \\
& \left.-4 M^{2}\left(\left|F_{n}\right|^{2}-\left|\overrightarrow{\mathrm{G}}_{n}\right|^{2}\right)\right\},
\end{aligned}
$$

where $e$ is the electronic charge. This result differs somewhat from that in Eq. (13) of Bethe ${ }^{7}$ as it contains the interference term between $F_{n}$ and $\vec{G}_{n}$ and has a different sign for the term $\left|\vec{p}+\vec{p}^{\prime} \cdot \vec{G}_{n}\right|^{2}$. In deriving Eq. (5) we use the relation

$$
q^{2}=\left(E-E^{\prime}\right)^{2}-\left(\overrightarrow{\mathrm{p}}-\overrightarrow{\mathrm{p}}^{\prime}\right)^{2},
$$

and we have omitted terms of the form $\left|\left(E-E^{\prime}\right) F_{n}-\left(\overrightarrow{\mathrm{p}}-\overrightarrow{\mathrm{p}}^{\prime}\right) \cdot \overrightarrow{\mathrm{G}}_{n}\right|^{2}$ since they vanish because of conservation of current. The matrix elements

$$
F_{n}=\left(n\left|e^{i \overrightarrow{\mathrm{q}} \cdot \overrightarrow{\mathrm{r}}}\right| 0\right)
$$

and

$$
\overrightarrow{\mathrm{G}}_{n}=\left(n\left|\vec{\alpha} e^{i \overrightarrow{\mathrm{q}} \cdot \overrightarrow{\mathrm{r}}}\right| 0\right)
$$

are calculated from the initial and final states $(0, n)$ and the Dirac matrix $\vec{\alpha}$ for the electron. For a many-electron target system, $F_{n}$ and $\vec{G}_{n}$ are summed over all electrons.

In the high- $Q$ approximation,

$$
\begin{aligned}
& \left|F_{n}\right|^{2}=\frac{1}{2} \frac{m}{Q+m} \frac{Q+2 m}{Q+M}, \\
& \left|\left(\overrightarrow{\mathrm{p}}+\overrightarrow{\mathrm{p}}^{\prime}\right) \cdot \overrightarrow{\mathrm{G}}_{n}\right|^{2}=\frac{1}{2} \frac{m}{Q+m} \frac{\left(\overrightarrow{\mathrm{p}}+\overrightarrow{\mathrm{p}}^{\prime}\right)^{2} Q}{Q+m},
\end{aligned}
$$

and

$$
\left|\overrightarrow{\mathrm{G}}_{n}\right|^{2}=\frac{1}{2} \frac{m}{Q+m} \frac{3 Q}{Q+m} .
$$

In Eqs. (9), (10), and (11), the factor $\frac{1}{2}$ comes from averaging over the spin of the electron in its initial state, and $m /(Q+m)$ comes from the normalization of the final-state wave function. Employing Eqs. (5) and (9) - (11), we can write the cross section for target electrons to appear in a given energy range $d Q$ at energy $Q$ for incident neutrinos of energy $E$. We obtain

$$
\begin{aligned}
d \sigma= & 4 \pi(e \lambda)^{2} \frac{m}{(Q+m)\left(E^{2}-M^{2}\right)} \\
& \times\left[E(E-Q)+\frac{Q M^{2}}{2 m}-M^{2}\right] \frac{d Q}{Q} .
\end{aligned}
$$

This result, which is exact within the high- $Q$ approximation, is similar to the cross section $d \sigma_{\text {Bethe }}$ given by Bethe's equation (21) of Ref. 7, but contains an additional term,

$$
d \sigma=d \sigma_{\text {Bethe }}\left(1+\frac{M^{2} Q}{2 m\left(E^{2}-E Q-M^{2}\right)}\right),
$$




$$
d \sigma_{\text {Bethe }}=4 \pi(e \lambda)^{2} \frac{m}{Q+m} \frac{E(E-Q)-M^{2}}{E^{2}-M^{2}} \frac{d Q}{Q} .
$$

When $Q<<E, M<<m$, and $M<<E$, the factor multiplying $d \sigma_{\text {Bethe }}$ in Eq. (13) approaches unity. The maximum energy $Q_{m}$ that can be transferred at energy $E$ is given by ${ }^{10}$

$$
Q_{m}=\frac{2 m\left(E^{2}-M^{2}\right)}{m^{2}+2 m E+M^{2}}
$$

We employ Eq. (12) to calculate the stopping power of massive neutrinos and apply it to study the solar neutrino anomaly.

\section{STOPPING POWER FORMULA AND THE SOLAR NEUTRINO ANOMALY}

The stopping power is given by

$$
-\frac{d E}{d s}=N \int_{\eta}^{Q_{m}} Q d \sigma,
$$

where $N$ is the number of electrons per unit volume in the medium and $\eta$ is the minimum energy lost in a collision. On substituting (12) in (16) and assuming that $\eta<<m$, we obtain

$$
-\frac{d E}{d s}=4 \pi N(e \lambda)^{2} \frac{m}{E^{2}-M^{2}}\left[\left(E^{2}+m E-\frac{3}{2} M^{2}\right) \ln \left(\frac{m+Q_{m}}{m}\right)-\left(E-\frac{M^{2}}{2 m}\right)\left(Q_{m}-\eta\right)\right] .
$$

Since the neutrino mass is small ( $<500 \mathrm{eV}$ ), we take $M<<m$ and $M<<E$. We express the neutrino magnetic moment in Bohr magnetons, i.e.,

$$
\lambda \equiv v \frac{e \hbar}{2 m c} \text {. }
$$

Reintroducing $c$ hereafter, we rewrite Eq. (17) as

$$
-\frac{d E}{d s}=\pi N\left(\frac{e^{2}}{m c^{2}}\right)^{2} v^{2} m c^{2}\left[\left(1+\frac{m c^{2}}{E}\right) \ln \left(\frac{m c^{2}+Q_{m}}{m c^{2}}\right)-\left(\frac{Q_{m}-\eta}{E}\right)\right] .
$$

For application to the neutrino anomaly, $\eta$ is approximately given by the plasmon energy of the solar electrons,

$$
\eta \sim \hbar\left(\frac{4 \pi N e^{2}}{m}\right)^{1 / 2} \sim 100 \mathrm{eV}
$$

with $N \sim 1.2 \times 10^{25} \mathrm{~cm}^{-3}$ being the solar electron density. A plot of $-\left(1 / v^{2}\right) d E / d s$ vs $E / m c^{2}$ is shown in Fig. 1.

Taking $-d E / d s$ as constant, we can calculate the energy lost by a neutrino which travels from the center of the sun to the surface by multiplying $-d E / d s$ with the radius of the sun, $7 \times 10^{10} \mathrm{~cm}$. When $v=10^{-9}, 3$ the energy lost by a $10-\mathrm{MeV}$ neutrino is found to be $\sim 2 \times 10^{-7} \mathrm{MeV}$, and $\sim 7 \times 10^{-8} \mathrm{MeV}$ for a $1-\mathrm{MeV}$. neutrino. By comparison, the energy loss of a $M=100-\mathrm{eV}$ neutrino in escaping the gravitational field of the sun is $\sim 2 \times 10^{-10} \mathrm{MeV}$. Consequently, neutrinos produced in the sun should escape from its surface with practically no energy loss owing to their magnetic-moment interaction with solar electrons. With previous investigators, ${ }^{6}$ we conclude that the electromagnetic interaction between neutrinos and electrons in the sun cannot be the dominant cause for the solar neutrino anomaly.

\section{TOTAL IONIZATION CROSS SECTION}

Integrating Eq. (12) from $Q=0$ to $Q_{m}$, Eq. (15), and assuming that $\eta<<m c^{2}$, we obtain the total cross section 


$$
\Phi=\pi\left(\frac{e^{2}}{m c^{2}}\right)^{2} v^{2}\left[\ln \left(\frac{Q_{m}}{\eta} \frac{m c^{2}}{m c^{2}+Q_{m}}\right)-\frac{2 E m c^{2}-M^{2} c^{4}}{2\left(E^{2}-M^{2} c^{4}\right)} \ln \left(\frac{m c^{2}+Q_{m}}{m c^{2}}\right)\right]
$$

On similar integration, Eq. (14) yields

$$
\Phi=\pi\left(\frac{e^{2}}{m c^{2}}\right)^{2} v^{2}\left[\ln \left(\frac{Q_{m}}{\eta} \frac{m c^{2}}{m c^{2}+Q_{m}}\right)-\frac{E m c^{2}}{E^{2}-M^{2} c^{4}} \ln \left(\frac{m c^{2}+Q_{m}}{m c^{2}}\right)\right]
$$

As $Q_{m}=E-m c^{2}$ when $M=m$ and $Q_{m}=2 E^{2} /\left(2 E+m c^{2}\right)$ when $M=0$, Eq. (22) reduces, respectively, to Bethe's equations (23) and (23a) in Ref. 7 for $M=m$ and $M=0$.

Asymptotically for $E>>m c^{2}$, Eqs. (21) and (22) become equal to the Bethe formula, viz.,

$$
\Phi=\pi\left(\frac{e^{2}}{m c^{2}}\right)^{2} v^{2} \ln \frac{m c^{2}}{\eta}
$$

\section{NEUTRINO MAGNETIC MOMENT}

An upper limit was calculated by Cowan and Reines $^{3}$ employing Bethe's formula, Eq. (14), and Muehlhause and Oleska's ${ }^{11}$ measured beta spectrum from fission as the antineutrino $\left(\bar{v}_{e}\right)$ spectrum. Recently the antineutrino spectrum was parametrized by Lee ${ }^{12,13}$ as

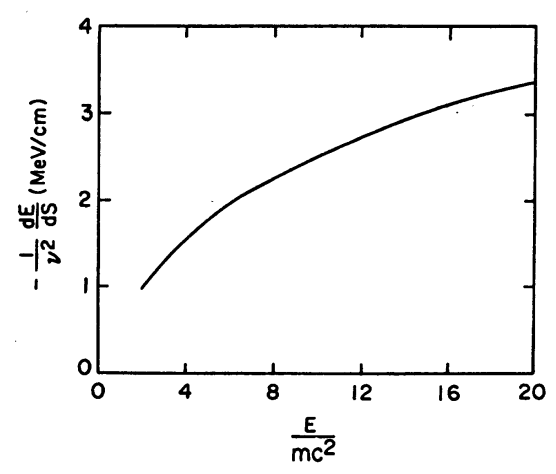

FIG. 1. Stellar neutrino stopping power in the reduced form $-\left(1 / v^{2}\right) d E / d s$ vs $E / m c^{2}$ for the solar electron density $N \sim 1.2 \times 10^{25} \mathrm{~cm}^{-3}$ and $m c^{2}=0.511 \mathrm{MeV}$.

$$
n(E)= \begin{cases}0, \quad E<0.055 \mathrm{MeV} \\ a \exp (-b E), & 0.055 \mathrm{MeV} \leq E \leq 3.4 \mathrm{MeV} \\ c \exp (-d E), & 3.4 \mathrm{MeV}<E\end{cases}
$$

where (in units of $\mathrm{MeV}^{-1}$ ) $a=3.63, b=0.543$, $c=17.8$, and $d=1.01$. Sobel et al. ${ }^{14}$ observed that the measured number of $\bar{v}_{e}$ in the energy range $E_{v}>4.5 \mathrm{MeV}$ is significantly less than that expected from the $\bar{v}_{e}$ spectrum of Avignone. ${ }^{15}$ These reductions were fitted by $L^{13} e^{13}$ with two sets of parameters representing the upper and lower limits of the measured number at $E=5.3 \mathrm{MeV}$. With this fit and Eq. (12), assuming $\mu / E<<1$, we can express the neutrino magnetic moment as

$$
v=0.877\left(\frac{S\left(Q_{2}, Q_{1}\right)}{G\left(Q_{2}, Q_{1}\right)}\right)^{1 / 2}
$$

where, as in Cowan and Reines, ${ }^{3} S\left(Q_{2}, Q_{1}\right)$ in the energy range $Q_{1}(0.1 \mathrm{MeV})$ to $Q_{2}(0.5 \mathrm{MeV})$ is the reactor associated counting rate for a detector containing $4 \times 10^{29}$ target electrons and $\bar{v}_{e}$ flux $F=1.3 \times 10^{13} / \mathrm{cm}^{2} \mathrm{sec}$. The largest counting rate found by Cowan and Reines was $2 \mathrm{sec}^{-1}$.

The quantity $G\left(Q_{2}, Q_{1}\right)$ is given by 
TABLE I. Parameters for $\bar{v}_{e}$ spectrum and results for the neutrino magnetic moment $v$ in units of the Bohr magneton.

\begin{tabular}{ccccccc}
\hline \hline $\begin{array}{c}\text { Parameter } \\
\text { set }\end{array}$ & $\begin{array}{c}a \\
\left(\mathrm{MeV}^{-1}\right)\end{array}$ & $\begin{array}{c}b \\
\left(\mathrm{MeV}^{-1}\right)\end{array}$ & $\begin{array}{c}c \\
\left(\mathrm{MeV}^{-1}\right)\end{array}$ & $\begin{array}{c}d \\
\left(\mathrm{MeV}^{-1}\right)\end{array}$ & $G\left(Q_{2}, Q_{1}\right)$ & $\begin{array}{c}v \\
(\mathrm{Bohr} \\
\text { magnetons })\end{array}$ \\
\hline No. $1^{\mathrm{a}}$ & 3.63 & 0.543 & 17.8 & 1.01 & 0.8130 & $1.38 \times 10^{-9}$ \\
No. 2 & 3.63 & 0.543 & 29.6 & 1.16 & 0.8100 & $1.38 \times 10^{-9}$ \\
No. 3 & 3.63 & 0.543 & 38.5 & 1.24 & 0.8086 & $1.38 \times 10^{-9}$ \\
\hline \hline
\end{tabular}

${ }^{\text {a Reference } 12 .}$

${ }^{\text {b Reference } 13 .}$

$$
G\left(Q_{2}, Q_{1}\right)=\frac{\int_{Q_{1}}^{Q_{2}} \frac{d Q}{Q} \frac{m c^{2}}{m c^{2}+Q} \int_{w}^{E_{\max }} n(E)\left(1-\frac{Q}{E}\right) d E}{\int_{0}^{E_{\max }} n(E) d E} .
$$

We calculated $G$ for the three sets of parameters published by Lee. ${ }^{12,13}$ The results for $v$ are listed in Table I. Evidently the neutrino magnetic moment upper limit, $v<1.4 \times 10^{-9}$ Bohr magneton, reported by Cowan and Reines is not altered by employing the $\bar{v}_{e}$ spectrum as parametrized by Lee.

\section{THERMALIZATION OF STELLAR NEUTRINOS}

Because of its possible cosmological implications, it is interesting to consider the distance over which stellar neutrinos thermalize as they traverse the known universe. Based on the estimated energy loss of $10^{-7} \mathrm{MeV}$ in the sun, which is a typical star, thermalization to stellar temperatures $\left(k T \sim 10^{-2} \mathrm{MeV}\right)$ would require a neutrino pass through $\sim 10^{5}$ stars. For an order of magnitude estimate of this likelihood we assume that there are $10^{11}$ galaxies in the universe, of radius $\sim 10^{10}$ light years $\sim 10^{28} \mathrm{~cm}$, with an average of $10^{12}$ stars per galaxy, each star being of the size of our sun. The implied average "macroscopic" mean free path between stellar encounters is $10^{40} \mathrm{~cm}$. The average number of stars traversed by a neutrino in traveling through the universe is then only $10^{-12}$, far below the needed $10^{5}$. We conclude that, as regards the interactions studied here, the thermalization of stellar neutrinos requires distances and times that are many orders of magnitude larger than the size and the age of the known universe. The energy of stellar neutrinos remains what it was at their creation. Energetic massive neutrinos are only weakly affected along their paths by gravitational interactions. Thus, in contrast to neutrinos created in the primordial universe, stellar neutrinos should not be clustered around galaxies but distributed uniformly throughout the universe.

\section{ACKNOWLEDGMENTS}

W. B. thanks Dr. N. Arista and Dr. F. Schulz for discussions during the early stages of this work. $\mathrm{He}$ is grateful for the hospitality extended him by Professor R. Sizmann of the Universität München under the auspicies of his Alexander-von-Humboldt Award, while this work was in progress. M. L. R. has benefitted from discussions with Professor B. P. Nigam. This research was supported in part by the Office of Health and Environmental Research, U. S. Department of Energy.
*Permanent address: Radiation and Solid State Laboratory, New York University, New York, N.Y. 10003.

1W. Pauli, in Rapport Septiene Conseil Physique, Solvay, Brussels, 1933 (Gautier-Villars, Paris, 1934).
2B. Kuchowicz, Rep. Prog. Phys. 39, 21 (1976).

${ }^{3}$ C. L. Cowan and F. Reines, Phys. Rev. 107, 528 (1957).

4J. Bernstein, M. Ruderman, and G. Feinberg, Phys. 
Rev. 132, 1227 (1963).

${ }^{5}$ D. Yu. Bardin, S. M. Bilenky, and B. Pontecorvo, Phys. Lett. 32B, 68 (1970).

6R. B. Clark and R. D. Pedigo, Phys. Rev. D $\underline{8}, 2261$ (1973).

${ }^{7}$ H. A. Bethe, Proc. Cambridge Philos. Soc. $\underline{31}, 108$ (1935).

8J. R. Oppenheimer and J. F. Carlson, Phys. Rev. 41, 763 (1932).

${ }^{9}$ W. Brandt, M. L. Rustgi, P. T. Leung, and J. E. Turner, Bull. Am. Phys. Soc. 26, 626 (1981).
${ }^{10}$ E. A. Uehling, Annu. Rev. Nucl. Sci. 4, 315 (1954).

${ }^{11}$ C. O. Muehlhause and S. Oleska, Phys. Rev. 105, 1332 (1957).

${ }^{12}$ H. C. Lee, Nucl. Phys. A $\underline{249}, 473$ (1978).

${ }^{13}$ H. C. Lee, Phys. Lett. 87B, 18 (1979).

${ }^{14}$ H. W. Sobel, H. S. Gurr, and F. Reines, in Unification of Elementary Forces and Gauge Theories, edited by D. B. Cline and F. E. Mills (Harwood, London, 1978), p. 111.

${ }^{15}$ F. T. Avignone, Phys. Rev. D 2, 2609 (1970). 